\title{
Track-etch membranes as templates enabled nano/micro technology : a review
}

\author{
S K Chakarvarti \\ Department of Applied Physics, National Institute of Technology (Deemed University), \\ Kurukshetra-136 119, Haryana, India \\ E-mail : skchakarvarti@nitkkr.ac.in
}

\begin{abstract}
Many techniques are being used in order to synthesize nano/micro materials falling under the realm of nanotechnology. It need not be overemphasized that the miniaturization of devices and synthesis of new materials have a tremendous role in the development of powerful electronics as well as material based technologies in other areas but for the laws of quantum mechanics posing limitations besides the increasing cost and difficulties in manufacturing in such a small scale. The quest, therefore, for the alternative technologies, have stimulated a surge of interest in nano-meter scale materials and devices in the recent years. Metallic as well as semiconducting nano wires are the most attractive materials because of their unique properties having myriad of applications like interconnects for nano-electronics, magnetic devices, chemical and biosensors, whereas the hollow tubules are equally considered to be candidates for more potent applications - both in physical as well as biosciences. Materials' processing for nano-structured devices is indispensable to their rational design. The technique, known as "Template Synthesis", using electrochemical/electro less deposition is one of the most important processes for manufacturing nano/micro structures, nano-composites and devices and is relatively inexpensive and simple. The technique involves using membranes - ion crafted ones (popularly known as Particle Track-Etch Membranes or Nuclear Track Filters), alumite substrate membranes, besides other types of membranes as templates. The parameters viz., diameter as well as length i.e., aspect ratio, shape and wall surface traits in these membranes are controllable.
\end{abstract}

In the present article a detailed review of this technique using track-etch membranes as templates in synthesis of nano/micro materials including hybrid materials and devices like field-ion emitters, resonant tunneling diodes (RTDs) etc. is presented including most of the results obtained in our laboratory.

Keywords : Nanotechnology, track-etch membrane, synthesis.

PACS Nos. : 85.35.-p, 81.07.-b

\section{Introduction}

Of late, there has been a tremendous growth of interest in potential applications of metallic as well as non-metallic nano/micro structures and materials. Nanotechnology has initiated a big leap and appears to be all set for bringing in revolution in the development and advancement of techniques involved in the synthesis and fabrication 
of sensors and devices. The conventional microelectronics fabrication techniques are facing harsh competitions from low dimensional strategies helping "Moore's Law" survive and cutting down the enormous costs. The conventional techniques for fabrication of very low dimensional wires - say quantum wires include wet chemistry, electron beam lithography, focused ion beam techniques and atomic-beam lithography [1] but for certain drawbacks and problems mentioned further. That has shown the ways for adopting newer alternative approaches which are relatively inexpensive, easier to handle and synergistically adorned with high efficacy. It is now well known that size of the devices and components dictate many unusual traits where quantum effects become more predominant. Quasi-one-dimensional nanostructures and materials like nano wires, fibres, tubules etc., having high aspect ratio would provide unusual and uncommon properties. Some properties like strength and hardness enhancement, dramatic changes in electrical (conduction, field-ion-emission through tunneling phenomenon, optical, magnetic, and chemical and other important functional attributes etc. are found to be enhanced when the size reduction comes into play. Materials with nanoscopic dimensions may exhibit quantized [2] conductance which not only has potential technological applications in various areas but also is of fundamental interest. This article addresses the art and science of specific technique - the "Template Synthesis" (TS) used as a route in the development of nano/micro materials, structures and devices rather than enumerating the detailed applications, properties and uses of such synthesized ensembles and materials. The recent past has witnessed keen interest being generated on the use of innovative technologies like TS in the production of nanomaterials' fabrication involving materials like metals, non-metals like semiconductors, magnetic multilayered nano wires, conducting polymers, glasses, nanotubues, wires and whiskers etc. reported from various authors and from our Lab [3-17].

\section{Methodology}

\subsection{Template synthesis - A technique :}

The technique of TS [18] may be classified into three categories depending upon the mode of use of templates [1]. The negative template method, positive template method and surface step-edge template method. The negative template methods, the most popular and widely used techniques of TS allow the use of prefabricated nanopores in solid templates and the material is deposited into these pores using normally electrochemical techniques. After removing (say by way of dissolving) the host template, free standing elements as wires, cylinders or conical structures can be obtained. The method has been regarded as "brute-force" method as the synthesized ensemble structure depicts the true replication of the morphology of the pores [19]. The fabrication of such templates involve many techniques including heavy ion irradiation of solid insulating materials like polymeric foils, micas, glasses etc. producing so called PTEMs. More details would follow later. The positive template methods use wire-like 
substrates like DNA [20], carbon nanotubes [21] etc. on the outer surface of which the material is deposited to the desired dimensions. The removal of templates can produce wire-like or tube-like structures. Other positive templates have been discussed by Huixin and Nongjian [1]. The surface Step-Edge templates, also known as "step-edges decoration" uses the fact that there is preferential or selective deposition of many materials initiating on the defect sites. Zach et al [22] and Bera et al [23] used this technique for generating conductive metal oxide $\left(\mathrm{MoO}_{\mathrm{x}}\right)$ at the step edges of $\mathrm{HOPG}$, which after reduction in appropriate environment could yield metallic Mo nano wires.

When elctrodeposition is carried out, the nucleation of nanostructures on the electrode substrate via template pores during electrodeposition is influenced by the crystal structure of the substrate, specific free energy and other factors like adhesion energy, orientation of the lattice of the substrate, etc. and the final size distribution of electrodeposits is strongly dependent upon the growth and nucleation kinetics [23]. Template assisted electrodeposition process can be divided into two categories : active template assisted process which results from growth of nuclei that essentially nucleate at the pores and defects of the substrate, while the other is known as restrictive template-based electrodeposition used mostly in the synthesis of metallic nano wires, involves deposition of metal into the prefabricated and designed pores within an inert, insulator membrane or template. PTEMs, porous alumina, conductive polymers, carbon etc. have been used as templates which fall under this category [23].

\subsubsection{Negative template synthesis :}

The basic and underlying principle of negative TS is similar to that of producing materials through the use of replication e.g., die-casting or mouldings like making icecandies. There are many methods used in practice for preparing nanomaterials - right from milling to lithographic techniques [24]. These techniques suffer from the problems like little control over the final morphology of the resulting nano-products, ensembles, and materials. The TS has in turn edge in this regard. It enables the synthesis of a variety of materials ranging from micro to nano dimensions, high aspect ratio and of desired morphology and geometry - the single unique attribute which makes this route as most acceptable and economical too. A template, in a general sense, may be defined as a pre-designed structure within which a network exists which can be utilized for further use. Thus, for example, a membrane with pre-fabricated cavities or pores of known morphology, number, distribution and configuration may also act as a template the pores of which can facilitate replication by any suitable means. Removal of the host template would lead to the presentation of ensembles whose morphological and stereo-chemical features and traits, might replicate the original cavities or pores in the template. Here in this technique, materials can be deposited within the cavities or pores or any other dimensional structures, by various methods like electrochemical or chemical reduction (electroless) of the appropriate ion. Depending upon the template 
geometries and the controlling factors, functional procedure and related parameters, the generated structures can be monodisperse homogeneous or heterogeneous, multilayered, short, squat fibrils, long needles, hollow tubules, tapered conical (single or double cones) elements etc. depending upon the template-factors stated above. The interesting aspect is one can have complete control over the aspect ratio (length and diameter ratio). Metallic as well as non-metallic tubules can be obtained by chemically derivating the pore/cavities walls by providing molecular anchors so that the electrodeposited metal deposits preferentially on the walls layer by layer, leading to hollow tubules when the process is terminated at a pre-estimated time interval [25]. The synthesized structures can remain either inside the host structures in the templates or they can be rendered free, and collected as ensemble. Alternatively, an ensemble of nano/microstructures that protrude from a surface like the bristles of a brush can be produced. The technique is blessed with simplicity and nano/microstructures with extraordinary low dimensions have been reported to be produced [26-28], which are otherwise difficult to manufacture using lithographic methods.

\subsection{History and development of negative template synthesis :}

Bean [29] first demonstrated the art of filling the pores of a membrane with silver followed by Possin [30] who utilized electrodeposition technique in the fabrication of thin wires as small as $400 \AA$ using mica with etched pores as templates for synthesis of such elements of the nanostructures. Williams and Giordano [31] claimed to have reduced the size down to $80 \AA$ after effecting some refinements to the technique. Penner and Martin [32] reported on the generation and characterization of ultramicroelectrodes with radii as small as $1000 \AA$. Klien et al [33] reported on the fabrication of graded $\mathrm{CdSe} / \mathrm{CdTe}$ hetero-structures and development of chemistry for fabricating II-VI chalcogenide semiconductors CdSe and CdTe within the template membranes producing micro-diode arrays consisting of micro-cylinders retinal rod cells - the photoreceptors in the human eye and having ca. five times smaller diameter than that of photoreceptors. Team led by Prof. Charles Martin at Colorado State University, USA, has been actively engaged in exploring exploiting the TS for its full potential and a large number of reports are available [34]. Researchers at GSI at Darmstadt, Germany (where this author has had also his first hand-on experience with the technique of TS for a short duration during early nineties), have also many reports to their credit for developing the technique further and generating nano/microstructures [35]. A large bulk of literature since then has been published on the negative TS and its applications $[3,34,36]$. This lab has also been reporting from time to time on the synthesis of nano/microstructures, devices and tubules using Particle Track Etch Membranes (PTEMs) as templates [3-17]. Almost simultaneously, the TS also included porous alumina $\left(\mathrm{Al}_{2} \mathrm{O}_{3}\right)$ membranes (which are prepared electrochemically from aluminum) (the fabrication method of anodic porous alumina started as early as 1950's) and have 
usually high pore density (as high as $10^{11} / \mathrm{cm}^{2}$ ) with pores as small as ca. $5 \mathrm{~nm}$, arranged in hexagonal arrays [37].

Here in the present work, detailed discussion has further been carried only on negative template synthesis using PTEMs as templates.

\subsection{Materials :}

While sieving properties of porous membranes have been used from as early as some thousand of years but it is relatively only recently that a technological application like TS of nano/microstructures has been brought to this process - almost a bi-product having no relation with sieving properties. Until recently where the use of positive template synthesis has started, most of the work had been carried out using negative template synthesis involving mainly two types of membranes - PTEMs and porous alumina $\left(\mathrm{Al}_{2} \mathrm{O}_{3}\right)$. Others include nanochannel array glasses, xeolite, proteins etc. $\mathrm{A}$ wide variety of other nanoporous solids that can be used as templates, are cited elsewhere [36].

\subsubsection{Particle Track Etch Membranes (PTEMs) :}

The most widely used materials in manufacturing PTEMs are polymers, micas and rarely this glass sheets. PTEMs, also known as Nuclear Track Filters (NTFs) have emerged as spin-off from Solid State Nuclear Track Detectors (SSNTDs) - solid dielectric materials capable of storing damage trails of energetic heavily ionizing ions which can subsequently be chemically amplified for observation as pores either through, say SEM or optical microscope [38-40]. The art and science used in crafting of PTEMs as templates involves two steps - irradiation of an SSNTD foil or a film by heavy energetic ion beam creating damage trails, also called as latent tracks, and followed by a controlled chemical etching of these latent tracks so as to produce see - through pores in the host material. The size and dimensions of the etched pores can be controlled conveniently through parametric control over the nature and energy of the intruding ions, the host material as target to be developed as template, chemical etch conditions viz., nature, concentration, temperature, agitation etc., besides preirradiation and post-irradiation storage conditions of the target material. The etched pores can have diameter ranging from few $\mathrm{nm}$ to $\mathrm{mm}$ [39]. NTFs have been put to numerous other applications besides their use as templates. There exists a wealth of literature on SSNTDs and related topics $[38,40]$. As discussed already, a large number of materials are used in manufacturing of NTFs which include mainly polymeric sheets, micas and glasses etc. The particle track-etch technique, therefore, enables the generation of pores of definite shapes which can either be used individually in the form of single particle track or collectively in the form of pore arrays constituting many pores - distributed either stochastically (pore density as high as $10^{10} / \mathrm{cm}^{2}$ ), or in a well defined spatially geometry through the control of the drilling particle beam used in 
"write mode". Areal dispersion usually lies between 2 to $20 \%$ arerkelq $\mathrm{MeV} /$ nucleon heavy ion (say, for example various projectiles used are collimated beam of fission fragments, heavy energetic ions like $\mathrm{U}, \mathrm{Pb}, \mathrm{Ag}, \mathrm{Si}, \mathrm{Bi}$, etc. whose range in the target material is far greater than the thickness of the target) has a range of the order of 10 micrometer in many polymers [39]. The custom made NTFs are available in a wide variety of pore size and porosities. The crafting of PTEMs templates through ions have been discussed in details $[38,39,41]$. Handled adequately and properly, these PTEMs can enable wires, tubules, solid cylinders, conical or tapered needles etc. with a well controlled dimension and shape besides a large aspect ratio. The dimensional parameters can vary from $10 \mathrm{~nm}$ to several micrometers with aspect ratio as high as 10-1000. The pores can be "drilled" with alignment in any direction depending upon the angle of incidence of the beam with the target. The synthesized members may either be left within the pores as embedded or can be removed either by dissolving or removing the host or pulling out mechanically but carefully of the host matrix. Figure 1 shows schematically the process of producing PTEM and its use as template and Figure 2 shows SEM photograph of a processed polycarbonate (Makrofol) NTF with monodisperse pores.

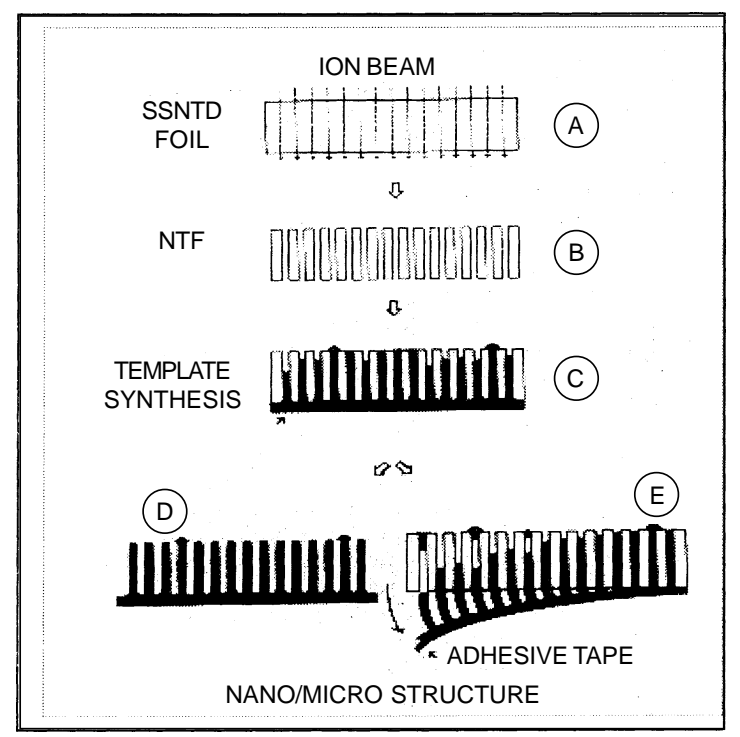

Figure 1. Schematic diagram showing production of a PTEM. NTF as a template for synthesis of low-dimensional template with monodispersed pores of dia structures. (A) Polymeric foil being irradiated with heavy ca. 1 micrometer and energetic ion beam, (B) Chemically etched foil as template or an NTF, (C) Deposited metal ions into the pores, producing filled pores, (D) Free standing structures after removal of host template and (E) Mechanical peelingan alternative to dissolution or removal of the host for retrieval of synthesized structures.

\subsection{Template synthesis of structures and devices:}

The strategy for embedding matter of interest within the etched pores or channels in the template is the material's placement through some suitable mechanism at the 


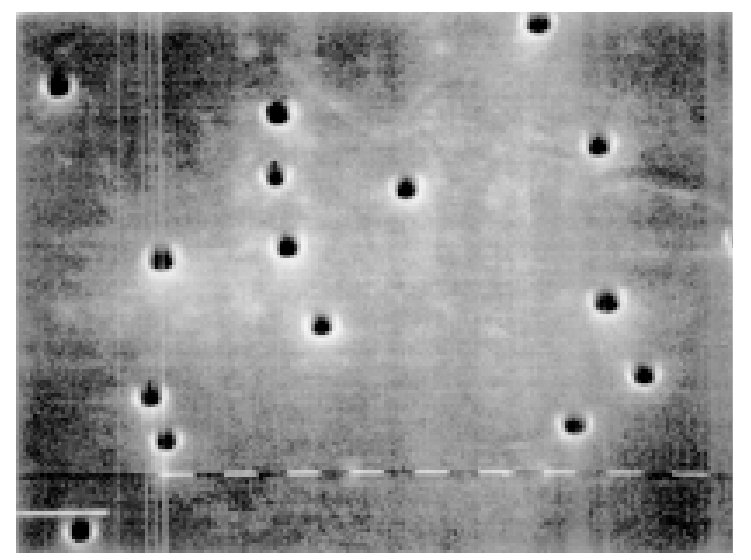

Figure 2. A processed polycarbonate (Makrofol).

desired places viz., pores. This can be accomplished in four ways. (1) Using the capillary action of the pores/channels and enabling the solution of the dissolved material to enter into the pores, followed by slow evaporation of the solvent. This will lead to growth of supersaturated structural elements. (2) Using electrochemical (galvanic) technique which is widely practiced. Here electrodeposition of the desired metal ions is carried out either using two-electrode or three-electrode electroplating cell [23]. (3) Using electroless (non-galvanic) deposition technique [42,43]. The underlying principle is to create nucleation centers on the pore walls and then precipitate the desired material on these nucleation centers. Through controlled manipulations, one can synthesize hollow as well as solid tubules. Metals like $\mathrm{Cu}, \mathrm{Ni}, \mathrm{Au}$, etc. or chalcogenides are dissolved in suitable solvents and precipitation can be facilitated on the pore walls. (4) Using chemical reactions, say, by permitting two reagents meet within the pores and react, produce precipitates, followed by drying process and their retrieval [10]. The necessary pre-requisite is that the penetrants acting as reactants must be hydrophilic otherwise one would require to apply hydrostatic pressure to forcibly inject them into the pores. In case of two gases (or one as gas and other participant as liquid) as reactants entering from both the faces of the template, the reaction can be accomplished within the pores [41].

In the proceeding paragraphs, more details of galvanic synthesis only are being provided. As mentioned, the technique is based upon the earlier work of Bean [29], Possin [30], Spohr [44], Williams and Giordano [31], Penner and Martin [32]. The simple underlying concept of electrodeposition of metals through electroplating is described as an electrochemical process in which metallic ions in supporting solution are reduced to the metallic state at the cathode, which, if closely covered by an NTF as a template and as an overlay, would lead to the formation of growth of plated film as the embodiment of micro- or nanostructure. The pores of the NTF used would act as template (Figure 1). 


\subsubsection{Electrochemical cells and electrodeposition :}

A scientific treatment of galvanic process is in fact complicated and development of optimum plating conditions may be obtained through one's personal experience obtained through repeated/trials under the given inputs. The age and condition of electrolyte viz., temperature, $\mathrm{pH}$, concentration, agitation rate, purity of solvent as well as electrolyte solute, additives and their amount, uneven current distribution, increase of specific resistivity of electrolyte, formation of gas bubbles and gas blankets at electrodes excessive inter electrode distance etc. affect the quality of deposition. Plating in small crevices or pores is very difficult. The solutions should have good micro-throwing powers - the ability of a solution to plate into fine cavities or defects on the surface such as pores, pits, polishing lines and scratches, besides the good covering power the ability of an electrolyte to deposit metals into pores where current density is low. Copper, for example, has poor macro-throwing power but can possess excellent microthrowing power. Electrodeposition of metals such as those which are more electronegative (e.g., aluminum, molybdenum, tungsten, titanium, tantalum, zirconium and niobium etc.) is not always possible from aqueous solutions. Electrodeposition on metals in corrosive electrolytes or galvanic deposition of noble metals such as gold and silver on base metals like copper needs much care as the adhesion of the plated mass is found to be poor. With micro porous membranes, rinsing off the membranes with $3 \% \mathrm{H}_{2} \mathrm{SO}_{4}$ followed by distilled water and absolute ethyl alcohol would facilitate the galvanic process in the case of metals like zinc, indium etc. [30]. Pre-soaking of the cleaned and washed template with the given electrolyte is also helpful [45]. Many detailed studies of the electrochemical fabrication process for nanostructures has been reported by many workers $[27,46]$.

For electrodeposition of metals, some special types of electro-plating cells are used $[32,45,47]$ which offer a low ohmic voltage drop across the electrodes even when the cell is filled with a poorly conducting electrolyte. There are various cell designs available - two electrodes and three electrode cells. The cell designed by Dobrev et al [47] has the provision for mechanical stirring besides a thermostat for maintaining the desired temperature of the water contained in a jacket surrounding the cell as constant. The Figure 3 shows the design of the two-electrode cell being used in this laboratory.

Electrodeposition is accomplished by simply coating one face of the overlaid template with a metal film (preferably gold) to serve as a working electrode on which electrodeposition takes place. The thickness of the film is appreciably large if the pores in the templates have also larger size. Alternatively, a metallic tape with conducting adhesive on the surface may also be used for fixing up the template membrane while the pores on the other face act as channel templates with one end sealed [45]. In order to make the pore walls conducting so as to be conducive in convenient electrodeposition by way of providing molecular anchors, the template may be moistened with solution like chloroplatinic acid [32] before electrodeposition is carried out. The use 
of electroless plating on the pore walls of polymeric templates might also be useful before galvanic process is started. This needs etching of polymer surfaces with chromic-sulphuric acid solutions followed by activation by immersion in tin and palladium solutions, or a colloidal solution containing these ions. This process is called "conditioning" which is required to improve the wettability of the polymer surface which further facilitates adhesion of the plated metal. During electrodeposition of the metal at the cathode, the depletion of the metal ions increases the absolute value of the deposition potential and if this exceeds the hydrogen over-voltage for the given process, hydrogen also deposits resulting into a loss of plating efficiency and increase in $\mathrm{pH}$ of the elctrodeposited metal, ultimately causing "burning" of the thin deposition. In order to avoid the development of over-potential, factors like increase in bath temperature and the agitation or the electrolyte are helpful. For applying deposition potential and monitoring the current and potential drop across the cell, a good constant voltage (potentiostat) power supply is needed.

It is found that while DC electrodeposition can produce good quality nano wires but the filling of the pores is partial ca. 10-20\% [48]. A high filling ratio as well as generation of uniform array of structures can be achieved by using AC electrodeposition. Various AC pulse shapes like saw-tooth waves, triangular, sine waves, but not square wave (square-wave potential pulses may be used for obtaining multilayered nano wires [49]) with varying frequencies have been used for better results, increased crystallinity and homogeneity [50]. Nielsch et al [51] used pulsed electrodeposition method and demonstrated that it suits for uniform deposition of porous alumina with almost $100 \%$ [51]. From our experience, we have found that initial high voltage for short duration followed by low values (the process is called "striking") produces good results. Chakarvarti and Vetter [3] have suggested a formula in order calculate an approximate optimum current for satisfactory results. It is of interest to note that the template synthesized materials (metals as well as semiconductors) in the form of solid cylinders, wires or whiskers can be crystalline [47,52].

\subsubsection{Synthesized structures and devices : some results :}

Some of the metallic, non-metallic homogeneous and heterogeneous structures and devices synthesized in this lab are shown in the Figures 3 to 15 . The template used in all cases were PTEMs of Makrofol polycarbonate having pores with different shapes (some cylindrical and others conical). The Makrofol foils were got irradiated at Gesellschaft fur Schwerionenforschung (GSI), Darmstadt, Germany. Also some polycarbonate foils were obtained from Whatman. More details and descriptions can be found from the references quoted.

Some of the nano/microdevices synthesized were resonant tunneling diodes (RTDs) made from heterojunctions of metal-semiconductors (Cu-Se, Zn-Se, Cd-Te systems [6,17], besides field-ion emitters [16,53]) using metallic nano/micro-tipped 


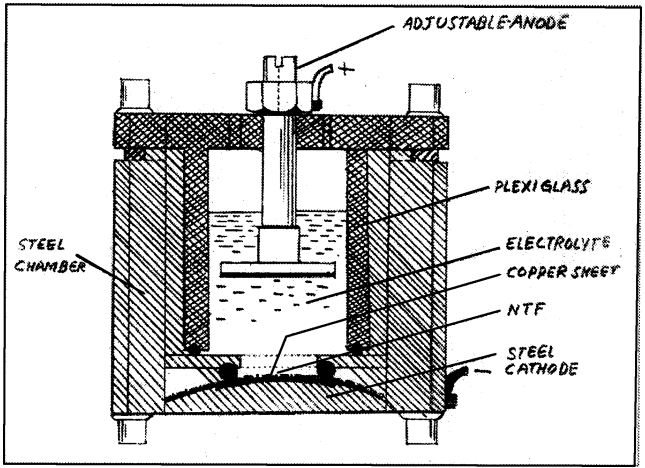

Figure 3. Design of two-electrode electrochemical cell used in template synthesis of nano/microstructures (3) PTEM as a template covers the cathode as an overlaywith the help of an "O" ring (3).

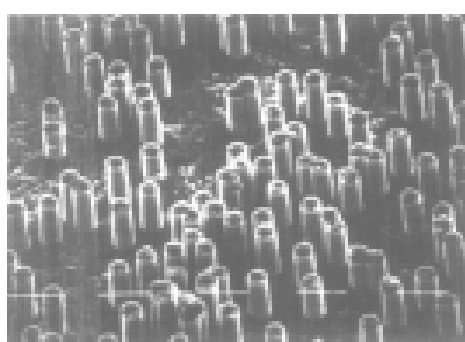

Figure 4. Copper microstructures (3).

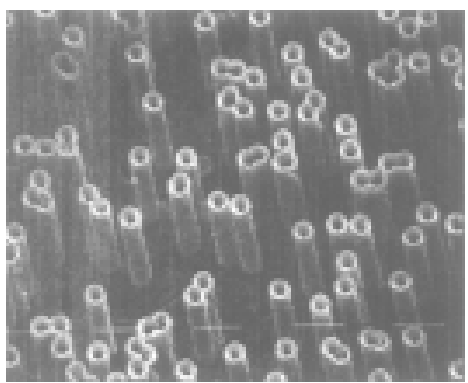

Figure 7. Copper hollow tubules.

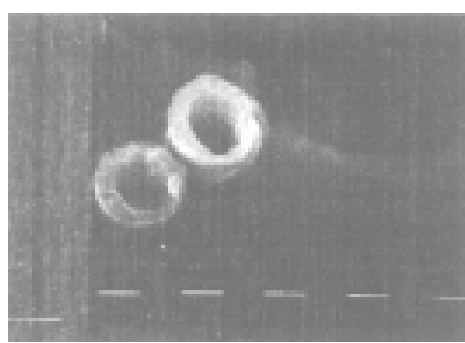

Figure 9a. Se microtubules (6).

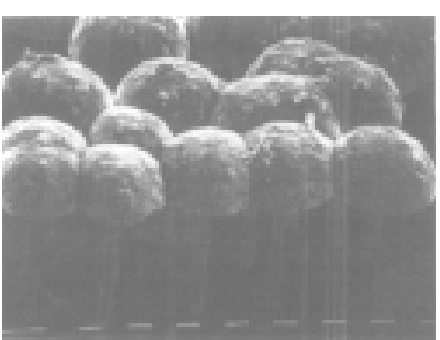

Figure 5. Sliver conical microstructures (16).

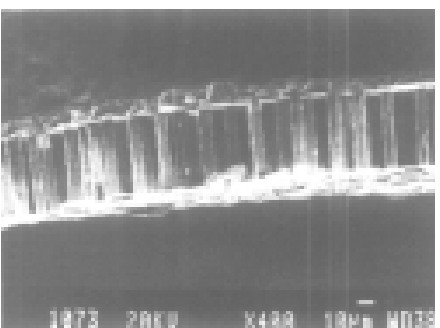

Figure 8a. Polypyrrole microtubules (11).

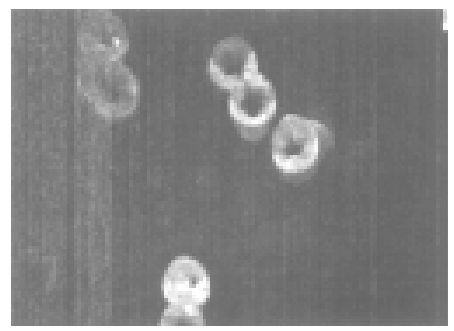

Figure 9b. Se microtubules (6).

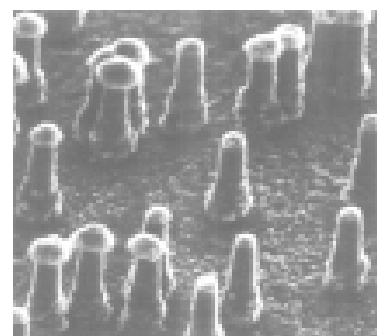

Figure 6. Buds and caps as over-deposition.

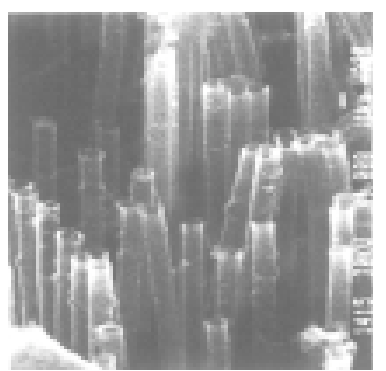

Figure 8 b. Thin walled polypyrrole tubules (11).

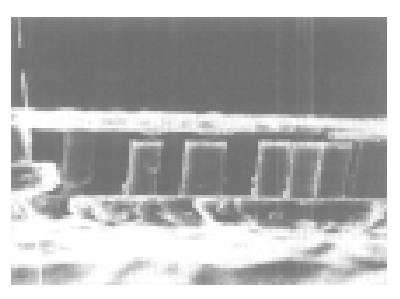

Figure 10. Cu-Se junctions (6). 


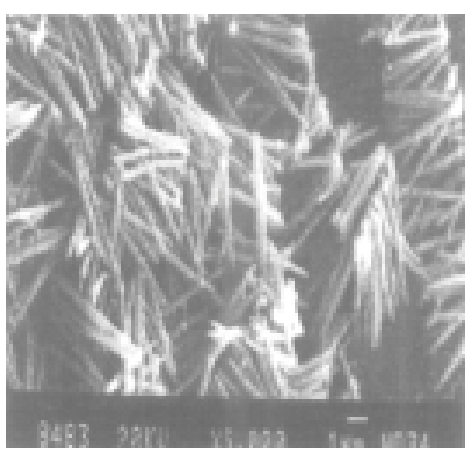

Figure 11. Cu-Se nano RTDs (53).

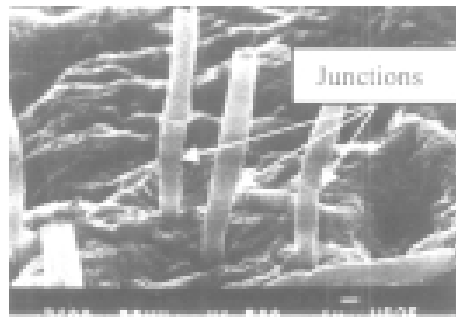

Figure 12. Zn-Se nano RTDs.

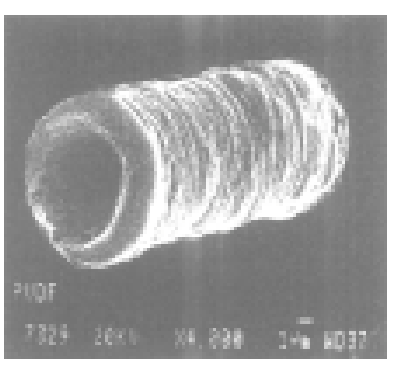

Figure 13. PVDF microtubule.

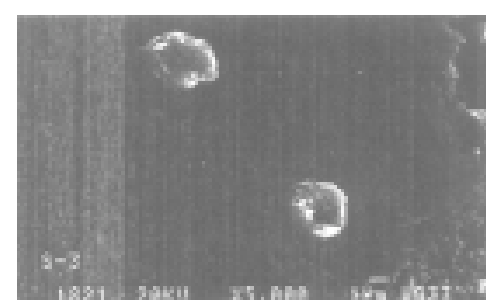

Figure 14. CdS microflowers (15).

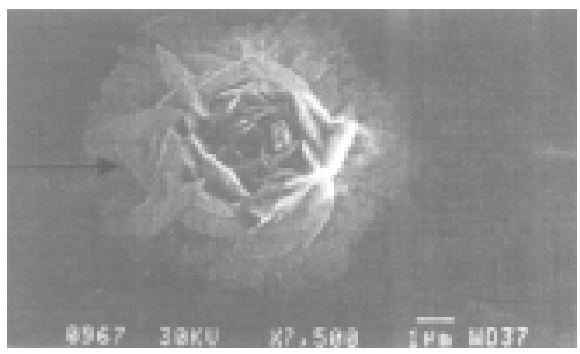

Figure 15. Copper microrose flower.

cylinders of copper and silver. For filling up the template pores, the electrodeposition was carried out from one face and the process was interrupted when the pores were filled half-way through. The electrolyte was replaced by another one with different metal/ semiconductor material composition and the deposition was further carried out till the pores are filled up to the brim. The Figure 16 shows typical I-V characteristics of nano

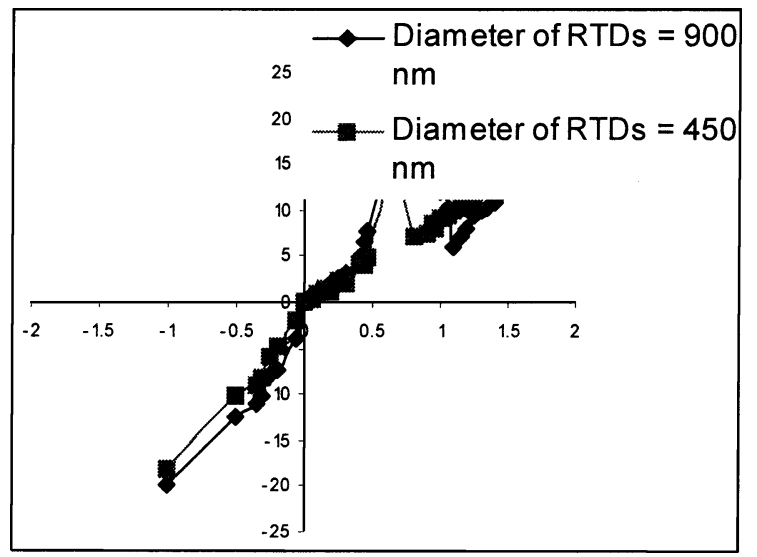

Figure 16. Collective current-voltage characteristics of Zn-Se RTDs with average diameters of 900 and $450 \mathrm{~nm}$. 
RTDs synthesized from Zn-Se deposition in Makrofol PTEMs. Confirming the fact that nature manifests identical both in small scale and macro scale as well. Figure 16 shows a floral display as a by-product of template synthesis process! More results on the effect of size as well as temperature of the synthesized RTDs of II-VI materials are on the way.

\section{Acknowledgments}

The author is grateful to Dr. Johann Vetter, Dr. Reimar Spohr, Dr. Ch. Trautmann and Dr. N Angert, Gesellschaft fur Schwerionenforschung (GSI), Darmstadt, Germany, for providing irradiation facility at their machine UNILAC. The thanks are also due to Director, National Institute of Technology (Deemed University), Kurukshetra, India for the financial help extended to the author under TEQIP to attend this Conference.

\section{References}

[1] Huixin He and Nongjian J Tao Encyclopedia of Nanoscience and Nanotechnology (ed.) H S Nalwa, Vol. 1 (USA : Academic Press) (2000)

[2] R Landauer J. Phys. Conden. Mat. 18089 (1989)

[3] S K Chakarvarti and J Vetter Radiat. Meas. 29149 (1998)

[4] C R Martin Chem. Mater. 81739 (1996)

[5] C J Brumlik, V P Menon and C Rmartin J. Mater. Res. 91174 (1994)

[6] S K Chakarvarti and J Vetter J. Micromech. Microengg. 357 (1993)

[7] Sanjeev Kumar, Shyam Kumar and S K Chakarvarti Current Sci. 87642 (2004)

[8] Sanjeev Kumar, Shyam Kumar and S K Chakarvarti J. Mat. Sci. Lett. 392951 (2004)

[9] Sanjeev Kumar, Shyam Kumar and S K Chakarvarti J. Mat. Sci. Lett. 393257 (2004)

[10] Sanjeev Kumar, Shyam Kumar and S K Chakarvarti Phys. Lett. A327 198 (2004)

[11] Sanjeev Kumar, D L Zagorski, Shyam Kumar and S K Chakarvarti J. Mat. Sci. Lett. 396137 (2004)

[12] Sanjeev Kumar, Shyam Kumar and S K Chakarvarti J. Optoelectronics and Advanced Materials 613 (2004)

[13] G S Sekhon, N K Verma, S K Chakarvarti and Sunil Kumar Atti Delia "Fondazione Giorgio Ronchi” 59529 (2004)

[14] Rajesh Kumar Vijay Kumar and S K Chakarvarti J. Mat. Sci. Lett. 403523 (2005)

[15] S K Chakarvarti Vijay Kumar and Sanjeev Kumar J. Mat. Sci. 40503 (2005)

[16] Rajesh Kumar and Shiv Kumar Chakarvarti Attidella "Fondazione Giorgio Ronchf 2005 LXI 31 (2006)

[17] Meeru Chaudhri, A Vohra, S K Chakarvarti and Rajesh Kumar J. Mat. Sci. : Materials in Electronics 17(12) 993 (2006)

[18] G A Ozin Adv. Mater. 4612 (1992)

[19] C A J Foss Metal Nanoparticles Synthesis, Characterization and Applications (USA : Marcel Dekker) (2002)

[20] E Braun, Y Eichen, U Salvan and G Ben-Yoseph Nature 391775 (1998)

[21] Sfullam, D Cottel, H Rensmo and D Fitzmaurice Adv. Mater. 121430 (2000)

[22] M P Zach, K H Ng and R M Penner Science 2902120 (2000)

[23] D Bera, S C Kuiry and S Seal JOM Jan (2004) http://www.tms.org/pubs/journals/JOM/0401/Bera-0401.html 
[24] G C Hhadjipanayis and R W Siegle Nanophase Materials : Synthesis, Properties, Applications Series E : Applied Sciences (Dordrecht) (1994)

[25] C R Martin, L S Van Dyke, Z Cai and W Liang J. Am. Chem. Soc. 1128976 (1990)

[26] R V Parthasarthy, K L N Phani and C R Martin Adv. Mater. 7896 (1995)

[27] C Schonenberger, B M I Vander Zande, L G J Fokkink, M Henny, C Schmid, M Kruger, A Bachtold, R Huber, H Birk and U Staufer J. Phys. Chem. B101 5407 (1997)

[28] M Sima, I Enculescu, C Trautmann and R Neumann J. Optoelectronics Adv. Mater. 6121 (2004)

[29] C P Bean, U S Patent No. 3483095 (1969)

[30] G E Possin Rev. Sci. Instrum. 41772 (1970)

[31] W D Willaims and N Giordano Rev. Sci. Instrum. 55410 (1984)

[32] R M Penner and C R Martin Anal. Chem. 592625 (1987)

[33] J D Klien, R D Herrick II, D Palmer, M J Sailor, C J Brumlik and C R Martin Chem. Mat. 5902 (1993)

[34] A Huczko Appl. Phys. A70 365 (2000)

[35] R Spohr Ion Tracks and Microtechnology : Basic Principles and applications (Vieweg : Wiesbaden) (1990)

[36] C R Martin Science 2661961 (1994)

[37] A Despic and V Parkhutik Modern Aspects of Electrochemistry (eds.) J O Bockris, R E White and B E (New York : Conway Plenum) (1989)

[38] R L Fleischer, P B Price and R M Walker Nuclear Tracks in Solids : Principles and Applications (Berkeley : Univ. of Calif. Press) (1975)

[39] B E Fischer and R Spohr Rev. Mod. Phys. 55907 (1983)

[40] P B Price Rad. Meas. 40146 (2005)

[41] E Ferain and R Legras Nucl. Instr. Meth. Phys. Res. B208 115 (2003)

[42] D Linkot, M Forment and H Cashet Adv. Electrochem. Sci. Eng. 6167 (1999)

[43] B Bercu, I Enculescu and R Spohr Nucl. Instrum. Meth. Phys. Res. B225 497 (2004)

[44] R Spohr US Patent No. 4338164 (1982)

[45] S K Chakarvarti and J Vetter Nucl. Instrum. Meth. Phys. Res. B62 109 (1991)

[46] T M Whitney, J S Jiang, P C Searson and C L Chien Science 2611316 (1993)

[47] D Dobrev, J Vetter and N Angert GSI Sci. Rep. 1994 (Darmstadt : Germany) (1995)

[48] A L Prieto, M S Sander, M S Martin-Gonzalez, R Gronsky, T Sands and A M Stay J. Am. Chem. Soc. 123 7160 (2001)

[49] L Piraux, J M George, J F Despres, C Leory, E Ferain and R Legras Appl. Phys. Lett. 652484 (1994)

[50] A J Yin, J Li, W Jian, A J Bennet and J M Xu Appl. Phys. Lett. 791039 (2001)

[51] K Nielsch, F Muller, A-P Li and U Gosele Adv. Mater. 12582 (2000)

[52] S K Chakarvarti, S Amrita Kaur and J K Quamara, Paper at Seminar on Current Developments in Disordered Materials (CDDM) (Kurukshetra University, Kurukshetra : India) (1996)

[53] Sanjeev Kumar, Rajesh Kumar, Shyam Kumar and S K Chakarvarti J. Mat. Sci. Lett. 40525 (2004) 